\title{
Usefulness of CT Scan in Diagnosing Acute Appendicitis in Patients with Low Alvarado Scores
}

\author{
Khawaja Bilal Waheed ${ }^{1}$, Waseem Jan Shah ${ }^{2}$, Ali Salman Alshehri ${ }^{3}$, Bilal Altaf ${ }^{4}$, Muhammad Amjad $^{5}$, \\ Muhammad Sohail Amin'6, Emad Fouad Mahmoud Said7, Noor Riyadh Bukhamsin ${ }^{8}$
}

\begin{abstract}
${ }^{1}$ Department of Radiology, KFMMC, Dhahran, Saudi Arabia. 2Department of Paediatric Surgery, KFMMC, Dhahran, Saudi Arabia. ${ }^{3}$ Department of Radiology, KFMMC, Dhahran, Saudi Arabia. ${ }^{4}$ Department of General Surgery, KFMMC, Dhahran, Saudi Arabia. ${ }^{5}$ Department of Gastroenterology, KFMMC, Dhahran, Saudi Arabia. ${ }^{6}$ Department of Radiology,

KFMMC, Dhahran, Saudi Arabia. ${ }^{7}$ Department of Radiology, KFMMC, Dhahran, Saudi Arabia. ${ }^{8}$ Department of Radiology, KFMMC, Dhahran, Saudi Arabia.
\end{abstract}

\section{ABSTRACT}

\section{BACKGROUND}

Alvarado score has been routinely used in clinical practice for assessing the probability of acute appendicitis in patients presenting with right lower quadrant abdominal pain. Computed tomography can be used for equivocal or difficult cases. We wanted to evaluate role of computed tomography (CT) in patients with low or equivocal Alvarado scores.

\section{METHODS}

This is a retrospective, observational study conducted in the Department of Radiology at our Hospital in Dhahran from October 2017 to October 2018. 138 patients with equivocal Alvarado scores (AS, 4-6) and negative (non-conclusive or limited) ultrasounds, were retrospectively reviewed. Pregnant, postoperative (bowel surgery) cases and chronic appendicitis cases were excluded. Diagnosis of acute appendicitis on CT was based on a thickened or fluid dilated appendix (diameter $>6 \mathrm{~mm}$, wall enhancement) with at least one of the associated findings (like appendicolith, peri-appendiceal fat stranding, free fluid, extra-luminal air foci, caecal base thickening and mesenteric lymph nodes). Imaging findings were reviewed by two radiologists and consensus reporting was made. Findings on CT were categorized as AA (Acute Appendicitis), OD (Other Diagnosis) and NOR (Normal). Surgical candidates were confirmed on histopathology, while nonsurgical cases were followed up on either imaging or clinical grounds. Chi-square test was used to determine association. The main outcome measure was diagnosis on CT and histopathology.

\section{RESULTS}

29.7\% (41 patients) were found to have acute appendicitis on CT imaging. Majority of patients with AS of 5 were having OD, while most of AA cases were having AS of 6 . All patients with AS of 4 were having normal CT scans.

\section{CONCLUSIONS}

Computed tomography helps to diagnose acute appendicitis or establish an alternate diagnosis in patients with equivocal Alvarado scores.

\section{KEY WORDS}

Computed Tomography, Alvarado Score, Acute Appendicitis
Corresponding Author: Khawaja Bilal Waheed, King Fahad Military Medical Complex, (KFMMC), P. O. Box 946, Postal Coad 31932, Dhahran, Saudi Arabia.

E-mail: khawaja@kfmmc.med.sa

DOI: $10.14260 /$ jemds/2019/653

Financial or Other Competing Interests: None.

How to Cite This Article: Waheed KB, Shah WJ, Alshehri AS, et al. Usefulness of CT scan in diagnosing acute appendicitis in patients with low alvarado scores. J. Evolution Med. Dent. Sci. 2019;8(40):3005-3009, $10.14260 /$ jemds/2019/653

Submission 09-08-2019, Peer Review 17-09-2019, Acceptance 25-09-2019, Published 07-10-2019. 


\section{BACKGROUND}

Lower abdominal pain is a common condition with wide range of causes. However, when it comes to right lower quadrant abdominal pain, acute appendicitis is undoubtedly the most common emergency condition requiring immediate management to avoid possible risks of perforation and peritonitis. ${ }^{1,2}$ Incidence of this condition seems to be stable in Western countries (between 6-9\%), but it appears to be rising in newly industrialized countries. ${ }^{3}$ Although the cause of such trend might be multifactorial, yet less consumption of dietary fibers has been theorized. ${ }^{3}$ It commonly occurs at early teens and late $40 \mathrm{~s}$ with slight male to female predominance, however, no age is spared. Various clinical scoring systems have been used in clinical practice for assessing probability of acute appendicitis. ${ }^{4}$ One of these, namely the Alvarado score (AS), was introduced in 1986,5 is based on history, clinical examination findings and a few laboratory tests. This scoring method has been widely assessed and utilized for evaluation of acute appendicitis to avoid negative appendicectomies. ${ }^{6}$ However, it is critical to understand applications and limitations of such methods. High AS scores (more than 6) in adult males are well validated while lower scores (less than 6) in other populations (like females and children) require clinical suspicion and often further investigation including radiologic imaging.6-8

Ultrasound is still important for an initial diagnostic work up patients with right lower quadrant pain, ${ }^{9}$ in particular for the paediatric age group and female patients, ${ }^{10,11}$ to avoid radiation risks and to exclude any gynaecologic cause in the later group. However, it is operator dependent. and can also be limited or technically difficult because of patient (large, fatty or obese) habitus or bowel gases. ${ }^{12}$ Advances in diagnostic imaging and early utility of computed tomography (CT) have reduced incidences of negative appendectomies. ${ }^{13}$ Many of the patients are found to have either mild degree of acute appendicitis or an alternate aetiology, guiding towards either a non-operative medical management (by observation and/or antibiotic course) or a specific treatment targeting an alternate diagnosis. However, radiation concerns with CT imaging are still to be considered with caution particularly in paediatric and female patients, ${ }^{14}$ where sometimes ultrasound or magnetic resonance imaging (MRI) might be a better option. Also, contrast related issues (like contrast reactions and contrast induced nephropathy) might be important limitation in few of vulnerable or elderly patients. ${ }^{15}$ Despite few reservations or contra-indications to CT imaging, it remains a crucial examination in difficult cases and in patients with equivocal AS.(4-6) Computed tomography has already shown to improve diagnostic yield in such patients with high sensitivity and specificity. ${ }^{16}$

Although various studies have documented validity of CT against higher AS,17 however, only a few have emphasized upon its prime utility in patients with low AS or equivocal cases that may end up in having aetiologies other than acute appendicitis. ${ }^{18}$ Prompt identification of an alternate diagnosis in such patients is strategic not only to reduce patient anxiety and hospital stay but also to guide clinicians towards specific treatment and avoid negative surgery (appendectomy). Therefore, we aim to highlight usefulness of CT imaging in such stratum of patients (i.e., with clinical suspicion of acute appendicitis and equivocal AS).

\section{METHODS}

This retrospective observational study was conducted in Radiology department at our Hospital in Dhahran from October 2017 to October 2018.

All patients $(\mathrm{N}=138)$ with equivocal clinical Alvarado scores (AS) between 4-6 on presentation, and initial negative (Non-conclusive, technically difficult or limited) ultrasound studies, were retrospectively reviewed for abdominal computed tomography (CT) findings. Pregnant patients and postoperative (bowel surgery or previous history of appendicectomy) cases were excluded. As the study was retrospective and did not involve disclosure of any patient information and privacy, the ethics committee of our Hospital waived the need for patient consent. The study was conducted in accordance with the Helsinki Declaration. Clinical information regarding Alvarado scores was obtained through initial assessment on patient's first presentation from clinical notes and Hospital Information System (HIS). Literature review was performed through electronic search (google scholar, pubmed).

Computed tomography examinations were performed on 128-slice scanner machine (SOMATOM Definition, Siemens Healthcare, Germany), acquiring portovenous phase of the abdomen and pelvis (from xiphisternum to symphysis pubis), after administration of intravenous contrast (Iomiron, GE Healthcare, Cork, Ireland). No oral contrast was given as per department protocol. Images were acquired in axial/ transverse planes, and reconstructed in coronal and sagittal planes.

Radiologic CT criteria for diagnosis of acute appendicitis was based on a thickened or fluid dilated appendix (of diameter more than $6 \mathrm{~mm}$ and wall enhancement) with presence of at least one of the associated findings (like appendicolith, peri-appendiceal fat stranding, free fluid, caecal base thickening and mesenteric lymph nodes). Imaging findings were reviewed by two radiologists (each having more than 7 years of body imaging experience) and final decision was made on consensus reporting.

Alvarado scores were obtained through the clinical notes at the time of presentation, that were based on the following method [Table 1]. Results of CT scans were categorized as having AA (Acute Appendicitis), OD (Other Diagnosis) and NOR (Normal) studies against Alvarado scores of 4 to 6.

\begin{tabular}{|c|c|}
\hline Symptoms & Score \\
\hline Migratory right iliac fossa pain & 1 \\
\hline Anorexia & 1 \\
\hline Nausea/Vomiting & 1 \\
\hline \multicolumn{2}{|c|}{ Signs } \\
\hline Tenderness at right iliac fossa \\
\hline Rebound tenderness \\
\hline Elevated temperature & 1 \\
\hline Laboratory Findings \\
\hline Leucocytosis & 2 \\
\hline Shift to the left neutrophils & 1 \\
\hline Total score & 10 \\
\hline Table 1. Alvarado Score for Clinical Assessment of Patients with \\
Right Lower Quadrant Abdominal Pain \\
\hline
\end{tabular}


Imaging diagnosed surgical cases were confirmed on histopathology, while non-surgical cases were confirmed on follow up imaging and stability (asymptomatic status) in next 3-6 months. The statistical analysis was carried out using Statistical Package for Social Sciences (SPSS, version 22). Chisquare test and t-test were used to determine association, and p-values less than 0.05 were considered significant.

\section{RESULTS}

Out of 138 patients, 74\% (53.6\%) were males and remaining $(\mathrm{N}=64,46.4 \%)$ were females, aged between $10-66$ years.

Computed tomography was able to indicate an abnormality in nearly $90 \%$ of the cases [table 2], and was found helpful in identifying difficult cases that were difficult to detect on ultrasound [fig. 1].

\begin{tabular}{|c|c|c|c|}
\hline \multicolumn{5}{|c|}{ Findings on Computed Tomography } \\
\hline Diagnoses & No. & Diagnoses & No. \\
\hline Acute appendicitis & 41 & Terminal ileitis & 07 \\
\hline Colitis (infectious) & 17 & Ruptured ovarian follicle/cyst & 09 \\
\hline Caecal diverticulitis & 03 & Pyelonephritis & 04 \\
\hline Ovarian cyst (complex) & 04 & Mesenteric lymphadenitis & 02 \\
\hline Ovarian torsion & 03 & Abdominal wall hernia & 03 \\
\hline Pelvic inflammatory disease & 08 & Foreign body ingestion & 01 \\
\hline Polycystic ovaries & 03 & Meckel's diverticulum & 01 \\
\hline Epiploic appendagitis & 04 & Neoplasm (colon, appendix) & 02 \\
\hline Omental infarction & 03 & Inflammatory bowel disease & 03 \\
\hline Ureteric stone & 05 & No abnormality detected & 15 \\
\hline Table 2. Findings on Computed Tomography in Patients \\
Presented with Right Lower Abdominal Quadrant Pain \\
\hline
\end{tabular}

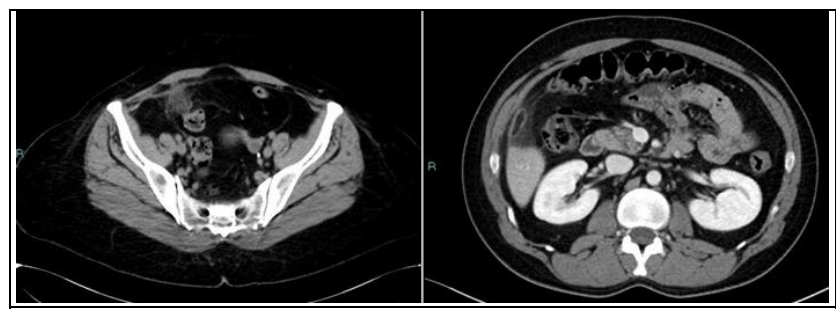

Figure 1. Two Different Patients of Epiploic Appendagitis, One with Inflamed Fat in Right Lower Abdomen Adjacent to Sigmoid Colon (Image on the Right), and other one Showing Inflamed Omental Fat at Right Subhepatic Region (Image on the Left). Note that this Entity along with Omental Infarction (Not Shown Here) are Sometimes Grouped Under Broader Umbrella Term of Intraperitoneal Focal Fat Infarction. Treatment is Medical, Unlike Acute Appendicitis.

\begin{tabular}{|c|c|c|}
\hline CT Findings & Frequency & Percentage \\
\hline AA & 41 & 29.7 \\
\hline NOR & 15 & 10.9 \\
\hline OD & 82 & 59.4 \\
\hline Total & 138 & 100.0 \\
\hline \multicolumn{2}{|c|}{ Table 3. Distribution of Patients on the Basis of their CT Diagnosis. } \\
AA-Acute Appendicitis, NOR- Normal, OD- Other Diagnosis \\
\hline
\end{tabular}

\begin{tabular}{|c|c|c|c|c|c|}
\hline \multicolumn{2}{|c|}{} & \multicolumn{3}{|c|}{ Alvarado Scores } & \multirow{2}{*}{ Total } \\
\cline { 3 - 5 } \multicolumn{2}{|c|}{} & $\mathbf{4 . 0}$ & $\mathbf{5 . 0}$ & $\mathbf{6 . 0}$ & n \\
\cline { 3 - 6 } & $\mathbf{n ( \% )}$ & $\mathbf{n ( \% )}$ & $\mathbf{n ( \% )}$ & $\mathbf{n ( \% )}$ \\
\hline \multirow{3}{*}{ Diagnosis } & AA & $24.9 \%$ & $1331.7 \%$ & $2663.4 \%$ & $41100.0 \%$ \\
\cline { 2 - 5 } & NOR & $15100.0 \%$ & $00.0 \%$ & $00.0 \%$ & $15100.0 \%$ \\
\cline { 2 - 5 } & OD & $33.7 \%$ & $5567.1 \%$ & $2429.3 \%$ & $82100.0 \%$ \\
\hline \multicolumn{2}{|c|}{ Total } & $2014.5 \%$ & $6849.3 \%$ & $5036.2 \%$ & $138100.0 \%$ \\
\hline
\end{tabular}

Table 4. Distribution of Patients by Alvarado Scores and CT Findings. AA-Acute Appendicitis, NOR- Normal, OD-Other Diagnosis

More than half (59.4\%) of patients were found to have OD on CT imaging while nearly one-third of patients (30\%) were having diagnosis of AA [table 3]. Common locations of appendix on CT were seen as retrocecal $(n=56,40 \%)$ and post-ileal $(n=39,28.2 \%)$, followed by less common sites in order as pelvic $(n=16)$, paracaecal $(n=15)$, promontric $(n=8)$ and subhepatic $(n=4)$. Out of 138 patients, $40 \%$ were having surgeries while $60 \%$ were non-surgical candidates. No negative appendectomies were seen. Most of patients with NOR, OD, and AA were having Alvarado scores of 4, 5, and 6 respectively [table 4], and these findings were found significant ( $\mathrm{p}$ value of 0.0005 ).

\section{DISCUSSION}

Acute appendicitis, a common surgical emergency, remains challenging in terms of diagnosis and management. Computed tomography has become the imaging choice in these patients particularly in adult male patients. ${ }^{19}$ Children, women and pregnant patients, for whom radiation risks need to be considered, another imaging (like ultrasound or MRI) remain an initial or sometimes the only appropriate option. ${ }^{15,20}$ However, if deemed necessary, a low dose-CT may be considered in these patients. ${ }^{16,18} \mathrm{CT}$ has shown to reduce negative appendectomies from $25 \%$ to $1-3 \%{ }^{17}$ Clinical Alvarado scoring is seen helpful to categorize patients for observation, imaging and surgery. Alvarado scores have been found to correlate well with the diagnostic screening and histopathologic findings. ${ }^{21}$ They found scores higher than 6 to be present with more advanced stages of acute appendicitis. We observed similar trend of such higher scores to be associated more with acute appendicitis on CT imaging. Many other studies have evaluated role of imaging compared to Alvarado scores, and demonstrated high Alvarado scores correlating well with imaging even in paediatric population, ${ }^{9}$ and pregnant women.22 However, the beneficial role of CT imaging in patients with equivocal AS scores, where an alternate diagnosis may be established and treated accordingly, is not well emphasized. We all have experienced a trend towards a more imaging based medical practice, possibly because of many reasons (Including availability of imaging techniques, patient satisfaction and better management), however, early utilization of imaging might be helpful in guiding clinicians towards prompt diagnosis and directed management avoiding prolonged hospital stay and patient anxiety.

We observed in our study that majority of patients (nearly $60 \%, \mathrm{n}=82$ ) were having AS of 5 , while less than a third of patients were diagnosed with acute appendicitis on CT imaging and most of these were having AS of 6 . Also, most of the patients with lower score (4) were NOR cases. These findings were found to be significant denoting importance of lower (4) and higher (6) values of equivocal AS and strategic role of CT imaging in establishing an alternate diagnosis in middle value of equivocal AS (i.e., 5) patients. Many of the studies have documented utilization AS, and compared its scoring with CT and surgical outcomes.23-25 Better clinical assessment and higher scores may help to avoid unnecessary CT examinations. ${ }^{25}$ However, this may not be true in difficult cases and further imaging may be warranted to establish a diagnosis. It should be noted that diagnosis of right lower abdominal quadrant pain may be quite challenging depending upon wide range of other aetiologies, and that too are different for different age groups. These may range from simple benign condition like mesenteric lymphadenitis in 
young children to worrisome colonic malignancy in elderly. Also, conditions related to urinary tract (ureteric stone), gallbladder (acute cholecystitis), omental fat (epiploic appendagitis, infarction), tubo-ovarian (Physiologic follicular rupture, ovarian stimulation, ovarian cyst, haemorrhagic cyst, torsion cyst, ectopic pregnancy, hydrosalpinx, complicated large fibroid or pelvic inflammatory disease), ileum (terminal ileitis, Crohn's disease, foreign body), colon (cecal diverticulitis, colitis or mass), and even retroperitoneum (psoas abscess, lipomatous mass) or bony abnormalities (Iliac bone osteomyelitis, bony tumour) may only be confirmed on further imaging. Except for bony abnormalities and ectopic gestation cases that were directly referred to orthopaedics and Ob-gynae respectively, we found nearly every aforementioned cause of OD in our study [table.2]. Although we did not include pregnant patients, however, we emphasize that MRI or low dose CT could be helpful in these cases depending upon the gestational stage if initial ultrasound remains non-conclusive. Poletti PA et al found that an algorithm integrating ultrasound and low dose CT was highly sensitive and specific for pregnant patients. ${ }^{20}$ Aguilera $\mathrm{F}$ found that MRI was very specific for acute appendicitis though with low sensitivity. ${ }^{14}$ Eng KA et al found in a metanalysis that second-line ultrasound, CT and MRI have comparable and high accuracies in helping to diagnose appendicitis in children and adults, including pregnant women. ${ }^{18}$ Esparaz JR et al results were in favour of utilization of CT and avoiding overuse of ultrasound in children with higher white blood cell counts and surgical consult. 15

We observed that our CT criteria for acute appendicitis was very helpful in determining acute appendicitis in all surgically proven cases. Different studies have documented similar CT criteria matching with the histopathologic findings of acute appendicitis by using combination of primary criteria of thickened or dilated appendix (diameter of more than $6 \mathrm{~mm}$ ) with wall enhancement, along with the secondary findings like presence of appendicolith, peri-appendiceal fat stranding, enlarged adjacent mesenteric lymph nodes, free fluid, and extra-luminal air foci, supportive of acute appendicitis. ${ }^{26-28}$ It should be noted that in our study the patients' referral for further imaging (In particular the CT scan) by the general surgeon (GS) rather than by the emergency room (ER) physician. Yazıcı $P$ et al found that AS of patients who were referred from GS for the CT imaging were having higher scores ( 5 or above) than those who were referred from the ER physician. ${ }^{13}$ They also observed that frequency of a negative CT was found higher with negative appendectomy, and a diagnosis of appendicitis with a rate of $25 \%$. In our study, we found that all patients with normal CT scans were having AS of 4 (15 patients), but one-fourth of patients with AS of 4 ( 5 out of 20 patients) were having OD (3 patients) and AA (2 patients). A retrocecal appendix and focal segmental or tip thickening were observed in those two positive cases. A negative CT was therefore found to be likely with lower AS (of 4), while a positive CT (For either AS or OD) more likely to be associated with higher AS scores (of 5 and 6).

Although we found lesser number of paediatric patients in our study ( 7 patients; 4 females, 3 males), aged between 10-14, yet we strongly recommend that CT should only be considered if clinical suspicion remains high and observation simply is not feasible (i.e., worsening of symptoms of AS), as we found either normal or benign findings (like mesenteric lymphadenitis or ruptured ovarian follicle) in most of these children. Therefore, in case of an initial limited, difficult or a non-conclusive ultrasound study, a low-dose CT or MRI may be considered in this age group to avoid radiation risks associated with CT imaging. Yazar AS and colleagues found a higher AS with positive ultrasonography in children with histopathologically proven acute appendicitis. ${ }^{9}$ Moreover, paediatric appendicitis score (PAS) may be used to estimate better clinical condition in these patients. ${ }^{29,30}$ Paediatric patients with a stable and low AS may benefit from simple observation after an initial negative ultrasound study, and may be offered a low-dose CT or MR imaging in case of worsening of AS.

Limitations to our study include single center, retrospective study not including chronic or complicated appendicitis cases (that might have a variable AS at presentation). Length of hospital stay was not documented, although patients with negative CT studies or benign findings (not requiring surgeries) presumably would have theoretically less hospital stays. Also, surgical cases were not categorized as open or laparoscopic procedures, as some of the patients might only had required diagnostic laparoscopy evaluation. Categories of OD were also not defined as surgical or non-surgical and benign or malignant, adding to important information set. Future studies adding these information sets on a larger scale would be of interest to provide further information for clinicians to adopt optimal diagnostic strategy and for directed patient management.

In summary, we conclude that based on our study results, CT scan after an initial negative or limited ultrasound study in patient with suspected acute appendicitis can be extremely helpful not only to diagnose acute appendicitis but also to establish an alternate diagnosis, particularly in patients with equivocal AS. Although CT may be time-consuming or impact resource utilization, however, it presumably reduces length of stay by avoiding prolonged observation in cases where a diagnosis cannot be ascertained clinically or remain difficult.

\section{CONCLUSIONS}

Computed tomography helps to identify a specific or an alternate diagnosis in patients with low or equivocal Alvarado scores.

\section{REFERENCES}

[1] Shogilev DJ, Duus N, Odom SR, et al. Diagnosing appendicitis: evidence-based review of the diagnostic approach in 2014. Western Journal of Emergency Medicine 2014;15(7):859-71.

[2] Wagner M, Tubre DJ, Asensio JA. Evolution and current trends in the management of acute appendicitis. Surg Clin North Am 2018;98(5):1005-23.

[3] Snyder MJ, Guthrie M, Cagle S. Acute appendicitis: efficient diagnosis and management. Am Fam Physician 2018;98(1):25-33. 
[4] Xingye W, Yuqiang L, Rong W, et al. Evaluation of diagnostic scores for acute appendicitis. J Coll Physicians Surg Pak 2018;28(2):110-4.

[5] Alvarado A. A practical score for the early diagnosis of acute appendicitis. Ann Emerg Med 1986;15(5):557-64.

[6] Kollár D, McCartan DP, Bourke M, et al. Predicting acute appendicitis? A comparison of the Alvarado score, the appendicitis inflammatory response score and clinical assessment. World J Surg 2015;39(1):104-9.

[7] Expert Panel on Gastrointestinal Imaging, Garcia EM, Camacho MA, et al. ACR Appropriateness Criteria ${ }^{\circledR}$ Right Lower Quadrant Pain-Suspected Appendicitis. J Am Coll Radiol 2018;15(11S):S373-S87.

[8] Lada PE. Alvarado score in the diagnostic of pain in the right lower quadrant. Rev Fac Cien Med Univ Nac Cordoba 2017;74(3):214-8.

[9] Yazar AS, Erdoğan S, Şahin C, et al. Reliability of ultrasonography and the Alvarado scoring system in acute appendicitis. Turk J Pediatr 2018;60(2):173-9.

[10] Kundiona I, Chihaka OB, Muguti GI. Negative appendicectomy: evaluation of ultrasonography and Alvarado score. Cent Afr J Med 2015;61(9-12):66-73.

[11] Jones RP, Jeffrey RB, Shah BR, et al. Journal Club: the Alvarado score as a method for reducing the number of CT studies when appendiceal ultrasound fails to visualize the appendix in adults. AJR Am J Roentgenol 2015;204(3):519-26.

[12] Pelin M, Paquette B, Revel L, et al. Acute appendicitis: factors associated with inconclusive ultrasound study and the need for additional computed tomography. Diagn Interv Imaging 2018;99(12):809-14.

[13] Yazıcı P, Öz A, Kartal K, et al. Emergency computed tomography for the diagnosis of acute appendicitis: How effectively we use it? Ulus Travma Acil Cerrahi Derg 2018;24(4):311-5.

[14] Aguilera F, Gilchrist BF, Farkas DT. Accuracy of MRI in diagnosing appendicitis during pregnancy. Am Surg 2018;84(8):1326-8.

[15] Esparaz JR, McGovern GC, Mowrer AR, et al. A simple algorithm to improve quality while reducing resource utilization in evaluation of suspected appendicitis in children. Am J Surg 2019;217(3):469-72.

[16] Tan WJ, Acharyya S, Goh YC, et al. Prospective comparison of the Alvarado score and CT scan in the evaluation of suspected appendicitis: a proposed algorithm to guide CT use. J Am Coll Surg 2015;220(2):218-24.

[17] Wang SY, Fang JF, Liao CH, et al. Prospective study of computed tomography in patients with suspected acute appendicitis and low Alvarado score. Am J Emerg Med 2012;30(8):1597-601.

[18] Eng KA, Abadeh A, Ligocki C, et al. Acute appendicitis: a meta-analysis of the diagnostic accuracy of US, CT and
MRI as second-line imaging tests after an initial US. Radiology 2018;288(3):717-27.

[19] Gorter RR, Eker HH, Gorter-Stam MA, et al. Diagnosis and management of acute appendicitis. EAES consensus development conference 2015. Surg Endosc 2016;30(11):4668-90.

[20] Poletti PA, Botsikas D, Becker M, et al. Suspicion of appendicitis in pregnant women: emergency evaluation by sonography and low-dose CT with oral contrast. Eur Radiol 2019;29(1):345-52.

[21] Do Nascimento RR, Souza JCG, Alexandre VB, et al. Association between the Alvarado score and surgical and histopathological findings in acute appendicitis. Rev Col Bras Cir 2018;45(5):e1901.

[22] Snyder MJ, Guthrie M, Cagle S. Acute appendicitis: efficient diagnosis and management. Am Fam Physician 2018;98(1):25-33.

[23] Al-Faouri AF, Ajarma KY, Al-Abbadi AM, et al. The Alvarado score versus computed tomography in the diagnosis of acute appendicitis: a prospective study. Med J Amed Forces India 2016;72(4):332-7.

[24] Chae MS, Hong CK, Ha YR, et al. Can clinical scoring systems improve the diagnostic accuracy in patients with suspected adult appendicitis and equivocal preoperative computed tomography findings? Clin Exp Emerg Med 2017;4(4):214-21.

[25] Apisarnthanarak P, Suvannarerg V, Pattaranutaporn P, et al. Alvarado score: Can it reduce unnecessary CT scans for evaluation of acute appendicitis? Am J Emerg Med 2015;33(2):266-70.

[26] Tatar IG, Yilmaz KB, Sahin A, et al. Evaluation of clinical Alvarado scoring system and CT criteria in the diagnosis of acute appendicitis. Article ID 9739385, Radiol Res \& Pract 2016;2016: p. 6.

[27] Suwal S, Karki S, Mandal D, et al. Multi-detector computed tomography evaluation of normal appendix. Kathmandu Univ Med J (KUMJ) 2016;14(56):357-61.

[28] Vaghela K, Shah B. Diagnosis of acute appendicitis using clinical Alvarado scoring system and computed tomography (CT) criteria in patients attending Gujarat Adani Institute of Medical Science - a retrospective study. Pol J Radiol 2017;82:726-30.

[29] Pogorelić Z, Rak S, Mrklić I, et al. Prospective validation of Alvarado score and pediatric appendicitis score for the diagnosis of acute appendicitis in children. Pediatr Emerg Care 2015;31(3):164-8.

[30] Díaz-Barrientos CZ, Aquino-González A, HerediaMontaño M, et al. The RIPASA score for the diagnosis of acute appendicitis: a comparison with the modified Alvarado score. Rev Gastroenterol Mex 2018;83(2):11216. 